\title{
INTRINSIC DISPERSION IN EARLY-TYPE STARS
}

\author{
Bengt STRÖMGREN \\ Institute for Avanced Study. Princeton
}

The principal basic parameters of early-type stars being mass and age, two-dimensional classification according to the MK system corresponds to a mass-age classification.

Other parameters which might vary from star to star include I. parameters characterizing the initial chemical composition, such as the initial helium-hydrogen ratio and heavy elementhydrogen ratio, 2. the angular momentum corresponding to the rotation of the star, and 3. a parameter, or parameters characterizing the magnetic properties of the star.

If in a group of stars only the parameters mass and age vary, then it must be possible to describe the entity of observable quantities accurately in terms of a two-dimensional classification. Any additional cosmical scatter indicates variations in other basic parameters from star to star.

I shall discuss here some results of photoelectric narrow-band photometry of early-type stars which have a bearing on the problem of cosmical scatter. The photoelectric photometry in question was carried out in collaboration with $\mathrm{D}$. L. Crawford with the 82-inch and 36 -inch reflectors of the Mc Donald Observatory.

Consider the two-dimensional classification of $\mathrm{O}$ and $\mathrm{B}$ stars based on the indices $\mathrm{l}$ and c determined through photoelectric narrow-band photometry (cf. B. Strömgren [I]). The index 1 measures the strength of $\mathrm{H} \beta$, the index $\mathrm{c}$ the Balmer discontinuity.

In the case of two-dimensional classification without cosmical scatter the absolute visual magnitude $M_{v}$ is expected to be uniquely determined by 1 and c. A test was carried out for member stars of the II Sco association for which absolute visual magnitudes of relatively high accuracy are available according to Blaauw and Bertiau. Spectroscopic binaries and non-members were eliminated as far as possible, and the (very small) scatter due to photometric errors was allowed for. The observations then indicate a small scatter in $M_{v}$ for equal 1 and $c$, measured by a probable error equal to $\pm 0^{\mathrm{m}} .22$.

Another test was carried out for members of the I Ori association. For the Outer nebula group considered by Blaauw [2] $\left(5^{\mathrm{h}} 27^{\mathrm{m}}\right.$ to $5^{\mathrm{h}} 35^{\mathrm{m}},-4^{\circ}$ to $-7^{\circ}$, excluding the stars in the region of the Orion nebula) a cosmical scatter in $\mathrm{M}_{\mathrm{v}}$ for equal 1 and $\mathrm{c}$ measured by a probable error $\pm \mathrm{o}^{\mathrm{m}}$. I6 was found.

The scatter in $\mathrm{M}_{\mathrm{v}}$ for equal $\mathrm{l}$ and $\mathrm{c}$ was investigated for the association I Lac on the basis of photometric observations made in collaboration with $\mathrm{D}$. L. Harris. It corresponds to a probable error of $\pm 0^{\mathrm{m}} \cdot 3 \mathrm{I}$. The question of membership of the association is somewhat more difficult to decide in this case than in the two previous cases.

If the stars of a group (a cluster or association) are assumed to be of practically the same age, then there should be a unique $\mathrm{c}-\mathrm{M}_{\mathrm{v}}$ relation, and a unique c-1 relation apart from the cosmical scatter which we are investigating.

The scatter in the $c-M_{v}$ relation for the stars of the Orion Outer nebula group considered above is easily derived from the UBV photometry of Sharpless [3]. It is found to be $\pm 0^{\mathrm{m}} .28$ 
in $\mathrm{M}_{\mathrm{v}}$ (for equal $\mathrm{c}$ or $(\mathrm{U}-\mathrm{B})_{\mathrm{o}}$ ) in the color range $(\mathrm{U}-\mathrm{B})_{\mathrm{o}}-\mathrm{O}^{\mathrm{m}} .2$ to $-\mathrm{O}^{\mathrm{m}} .8$. Part of this scatter may be due to undetected spectroscopic binaries.

On the basis of the c-l photometry by Crawford and myself I have derived the following scatter (p.e.) in the c-l relation for II Sco and I Ori : $\pm \mathrm{o}^{\mathrm{m}} .005$ in 1 for equal c, for the (U-B)o range $-0^{\mathrm{m}} .2$ to $-\mathrm{I}^{\mathrm{m}} .0$. The l-scale is such that this corresponds to a scatter (p.e.) of $\pm 0.35 \AA$ in the equivalent width of $\mathrm{H} \beta$ for equal $\mathrm{c}$. The contribution of errors in the photometry is small and has been allowed for. It should be noted that the effect of undetected spectroscopic binaries is small in this case.

The cosmical scatter corresponding to variations in parameters other than mass and age in the cases just discussed referred to members of the same association. It is of great interest to compare different associations. Consider the average curve of the main-sequence B stars of an association in the l-c diagram. In some cases it can be concluded from the presence of very luminous main-sequence stars that the age of the association is small enough for the l-c line of the association to correspond to the age-zero line, at least for the spectral classes B 6 B 9, say. If the stars of two associations do not differ systematically in initial chemical composition (or with respect to properties of rotation and magnetic fields), then we expect their agezero l-c lines to coincide.

The I Ori and II Sco association have been compared in this way. It was found that the two average $1-\mathrm{c}$ lines coincide within $0^{\mathrm{m}} .003$ in 1 for equal $\mathrm{c}$.

If we interpret differences in the 1-c lines in terms of differences in the initial heliumhydrogen ratio, then we find in the particular case of the comparison of I Ori and II Sco that the initial relative hydrogen content $X$ cannot differ by more than I0-20 per cent.

It would be of considerable interest to apply this procedure to a number of associations, located in more than one spiral arm, and if possible also in other galaxies. In this connection it should be noted that the photoelectric narrow-band photometry in question can be carried out without any difficulty for relatively faint stars (cf. [I]).

R E F E R E N C E S

[1] B. Strömgren, Pontificial Academy, Stellar Populations ed. O'Connell, 245, North Holland Publishing Co., I958; Specola Vaticana, 1958.

[2] A. Blaauw, Charlottesville Conference on the Cosmic Distance Scale, A. J., 63, 186, 1958.

[3] S. Sharpless, Ap. J. 116, 25I, 1952, 119, 200, I954. 